\title{
Técnicas de pré-preparo de feijões em unidades produtoras de refeições das regiões Sul e Sudeste do Brasil ${ }^{1}$
}

\author{
Bean prepreparation techniques used by \\ foodservices in Southern and \\ Southeastern Brazil
}

Ana Carolina FERNANDES²

Maria Cristina Marino CALVO2

Rossana Pacheco da Costa PROENÇA²

\section{R E S U M O}

\section{Objetivo}

Considerando a importância da alimentação fora de casa nos hábitos contemporâneos e o papel cultural e nutricional do feijão na alimentação brasileira, este estudo objetivou investigar as técnicas de pré-preparo de feijão utilizadas nas Unidades Produtoras de Refeições das regiões Sul e Sudeste do Brasil.

\section{Métodos}

Foi elaborado um questionário, disponibilizado online. A amostra foi composta por nutricionistas responsáveis técnicos de Unidades Produtoras de Refeições e definida por saturação de tempo: o questionário esteve disponível na web por dezoito semanas. O questionário continha sistema de identificação por número do registro no respectivo órgão profissional fiscalizador de sua região, o que evitou duplas respostas e possibilitou a limitação por região do País. Os dados foram analisados por estatística descritiva básica e teste de Qui-quadrado.

\section{Resultados}

Registraram-se 445 respostas de nutricionistas responsáveis por 413688 refeições diárias. Quarenta e nove por cento da amostra relataram realizar remolho do feijão. Não foi encontrada associação entre realização de remolho, tipo de feijão, número de refeições servidas ou localização geográfica da unidade produtora de refeições. Dentre as justificativas para realização de remolho do feijão, as questões operacionais sobressaíram-se às sensoriais, e, em 69\% dessas Unidades Produtoras de Refeições, a água de remolho é descartada antes da cocção.

\footnotetext{
1 Artigo elaborado a partir da dissertação de AC FERNANDES, intitulada "Técnicas de preparo e tipos de feijão utilizados em Unidades Produtoras de Refeições das regiões Sul e Sudeste do Brasil”. Universidade Federal de Santa Catarina; 2010.

2 Universidade Federal de Santa Catarina, Centro de Ciências da Saúde, Departamento de Nutrição, Programa de Pós-Graduação em Nutrição. Campus Trindade, 88040-970, Florianópolis, SC, Brasil. Correspondência para/Correspondence to: RPC PROENÇA. E-mail: <rossana@mboxl.ufsc.br>.
} 


\section{Conclusão}

Observou-se que a realização de remolho do feijão parece ser determinada pela percepção do nutricionista sobre sua importância, o que pode ser um reflexo da escassez de discussões quanto à influência das técnicas de preparo na qualidade nutricional e sensorial desse alimento.

Termos de indexação: Água. Phaseolus. Questionário. Serviços de Alimentação.

\section{A B S T R A C T}

\section{Objective}

The importance of food away from home nowadays and the cultural and nutritional role of beans in the Brazilian diet led this study to investigate the bean prepreparation techniques used by foodservices in the Brazilian South and Southeast regions.

\section{Methods}

A questionnaire was developed and made available online. The sample consisted of dieticians in charge of foodservices and sample size was time-dependent: the questionnaire was available online for 18 weeks. The questionnaires included dieticians' professional license numbers to prevent repeated answers. The data were analyzed by basic descriptive statistics and the chi-square test.

\section{Results}

A total of 445 dieticians responsible for 413,688 meals a day answered the questionnaire. Nearly half the sample (49\%) soaked the beans before preparation. There was no association between soaking and type of bean, number of meals served per day or foodservice location. Beans were usually soaked for operational reasons rather than sensory reasons. Most foodservices (69\%) discarded the soaking water.

\section{Conclusion}

Apparently, the decision to soak depends on the dietician's perceived importance of soaking, which may be attributed to the scarcity of information about the effect of soaking on the sensory and nutritional quality of this food.

Indexing terms: Water. Phaseolus. Questionnaire. Food Services.

\section{N T R O D U Ç Ã O}

O feijão é consumido no mundo todo, principalmente na América Latina, na Índia e na África. No Brasil, o feijão faz parte da alimentação cotidiana do brasileiro, tendo se tornado parte da cultura alimentar logo após a chegada dos portugueses, no século XVI'1.

No ano de 2003, o Brasil foi o quinto maior consumidor mundial de feijão per capita². Entretanto, conforme dados do Instituto Brasileiro de Geografia e Estatística (IBGE) ${ }^{3}$, entre os anos de 1975 e 2002, houve uma queda de $30 \%$ na participação do feijão e de outras leguminosas na dieta do brasileiro.

Comparando-se os dados da Organização das Nações Unidas para Agricultura e Alimentação (FAO), que incluem o consumo de feijão fora de domicílio, e os dados do IBGE, que não o inclui, deduz-se que $27 \%$ do feijão no Brasil são consumidos fora do lar, em Unidades Produtoras de Refeições (UPR) comerciais ou coletivas ${ }^{4}$.

Diante do atual panorama de consumo de feijão no Brasil e da importância nutricional e cultural desse alimento no País, o Guia Alimentar para a População Brasileira recomenda o consumo de pelo menos uma porção de feijão por dia, variando-se os tipos de feijão, como o preto, carioquinha, vermelho, entre outros ${ }^{5}$.

Dentre as vantagens do consumo dessa leguminosa descata-se seu efeito hipocolesterolêmico ${ }^{6,7}$, sua baixa quantidade de gordura e o elevado conteúdo de carboidratos complexos, proteínas, vitaminas do complexo B, ferro, cálcio e fibra alimentar ${ }^{5}$. 
Entretanto, as sementes das leguminosas contêm fatores antinutricionais, como os inibidores de tripsina, os fitatos, os polifenóis (nos feijões, principalmente os taninos) e os oligossacarídeos não digeríveis. Alguns são termolábeis, desaparecendo depois de cozimento adequado. Outros, embora termoestáveis, podem ter suas concentrações reduzidas por dissolução na água ${ }^{8}$.

O remolho prévio do feijão em água e o posterior descarte da água podem vir a diminuir a quantidade desses compostos. Alguns estudos obtiveram uma maior redução do teor de taninos e fitatos em feijões deixados de remolhos e cozidos sem a água de remolho-11.

O procedimento de remolho do feijão durante o seu pré-preparo parece ser unanimemente recomendado pelos cientistas; porém, não há um consenso quanto ao tempo de remolho nem quanto à necessidade de eliminação ou não da água'12.

Nesse contexto, o presente estudo objetivou identificar as técnicas de preparo de feijões comuns (Phaseolus vulgaris L.) utilizadas em Unidades Produtoras de Refeições das regiões Sul e Sudeste do Brasil, e analisar também os motivos para essas escolhas.

\section{MÉ T O D O S}

Realizou-se um estudo de caráter exploratório, cuja população foi composta pelos nutricionistas responsáveis técnicos de Unidades Produtoras de Refeições comerciais e coletivas das regiões Sul e Sudeste do Brasil.

A pesquisa foi realizada com nutricionistas visto que, de acordo com a Lei brasileira de $n^{\circ}$ $8.234^{13}$, as atividades de planejamento, organização, direção, supervisão e avaliação de serviços de alimentação e nutrição são privativas desse profissional. Além disso, com a escolha de uma classe profissional específica, busca-se obter uma maior homogeneidade das características da amostra e diminuir os vises de resposta que po- deriam ser causados por diferenças no nível de escolaridade, conhecimentos técnicos e poder de decisão nas UPR.

A escolha pelas regiões Sul e Sudeste deve-se a semelhanças no que se refere aos tipos de feijão comuns - Phaseolus vulgaris L. - predominantemente consumidos, eliminando-se locais onde o consumo de outras espécies de feijão, como o feijão-de-corda (Vigna unguiculata (L.) Walp), é dominante ou expressivo ${ }^{14}$.

\section{Elaboração do questionário}

Conforme os objetivos do estudo, foram propostos os temas das perguntas que compuseram o questionário aplicado aos nutricionistas responsáveis técnicos (Figura 1).

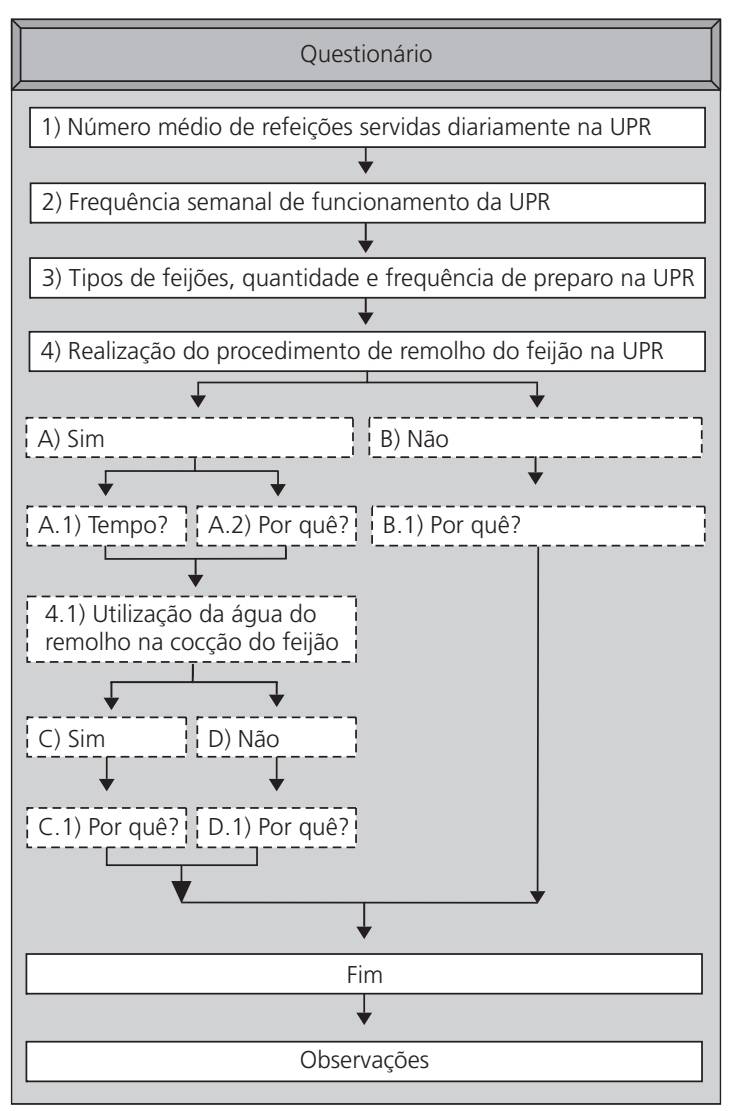

Figura 1. Esquema de estruturação do questionário. Florianópolis (SC), 2010. 
O questionário foi composto de uma questão aberta (questão 1), de questões fechadas com escolha única (questões $2,4,4.1$ ) e de questões mistas, contendo respostas fechadas de múltipla escolha e a opção "outros", para resposta aberta (questões 3, A.1, A.2, B.1, C.1, D.1).

Depois de elaboradas as perguntas e as respostas fechadas, o questionário ficou disponível online, durante um mês, para realização de um estudo-piloto com formandos do curso de graduação em nutrição e mestrandos nutricionistas do Programa de Pós-Graduação em Nutrição da Universidade Federal de Santa Catarina. Com base nas sugestões dos participantes, foram realizados ajustes no questionário, cuja versão final foi publicada para coleta de dados.

A aplicação do questionário foi feita pela página da web, com a divulgação por correio eletrônico. Esse método é comparável aos procedimentos utilizados pela via postal, porém com as vantagens do baixo custo, da praticidade, da rapidez, dos recursos de multimídia, da interatividade, da possibilidade de envio automático das informações ao banco de dados e da pronta análise ${ }^{15}$.

Os inquéritos feitos pela Internet podem ter limitações quanto à identificação por endereço eletrônico, pois um sujeito pode ter e utilizar mais de um endereço ${ }^{15}$. Visando a minimizar essa possibilidade, definiu-se que a identificação dos respondentes seria realizada por número de registro no respectivo órgão profissional fiscalizador de sua região, o Conselho Regional de Nutricionistas (CRN).

Além de evitar duplas respostas e aumentar a probabilidade de retornos confiáveis e provenientes somente do público-alvo, foi possível descartar os profissionais com registro fora da área de interesse da pesquisa pela identificação do primeiro número do registro, que indica a região ou o estado do CRN equivalente.

Os conselhos equivalentes aos estados do Sul e Sudeste do Brasil são o CRN-2, de Santa
Catarina e Rio Grande do Sul; o CRN-3, de São Paulo(3); o CRN-4, do Rio de Janeiro e Espírito Santo; o CRN-8, do Paraná, e o CRN-9, de Minas Gerais.

\section{Disponibilização e divulgação do questionário}

O questionário online com as perguntas definitivas foi publicado em junho de 2009, com divulgação simultânea pelos Conselhos Regionais de Nutrição (CRN). A divulgação via correio eletrônico também foi feita junto a associações e sindicatos de nutricionistas, instituições com cursos de graduação e de pós-graduação em nutrição, a empresas concessionárias de alimentação coletiva e outras UPR, bem como às redes pessoais e profissionais de contato dos pesquisadores envolvidos neste estudo.

Ademais, fez-se a divulgação da pesquisa em eventos de Nutrição, por meio de cartazes, da entrega de panfletos e da exposição oral ao final de palestras, cursos e apresentações de temas livres conduzidos pelo núcleo de pesquisa envolvido no projeto.

Visando a aumentar a taxa de resposta dos questionários, foi definida uma periodicidade para envio de lembretes que ressaltavam a importância dos resultados da pesquisa e reforçavam o pedido para preenchimento do questionário, com base em metodologias de inquéritos postais, uma vez que não foram encontradas recomendações de envio de lembretes com relação à pesquisa via internet. Foi adotado o padrão de periodicidade de lembretes adaptado de Rea \& Parker $^{16}$, que sugerem, no caso de inquéritos postais com prazo maior que duas semanas para resposta, que devam ser mandados dois lembretes de resposta: o primeiro após seis semanas e o segundo depois de oito semanas do envio inicial.

Em virtude de o período de disponibilização do questionário ser de dezoito semanas,

\footnotetext{
3 O CRN-3 abrange também o Mato Grosso do Sul, que faz parte da região Centro-Oeste. Visando evitar respostas não desejadas no questionário online, questionou-se o estado de atuação dos profissionais da região 3, aceitando-se apenas as respostas daqueles que atuavam em São Paulo.
} 
adaptou-se o intervalo de tempo sugerido para o envio de lembretes, aumentando-o para oito semanas até se remeter o primeiro lembrete e mais seis semanas até a emissão do segundo, seguido de quatro semanas até o encerramento do período de coleta de dados.

O tamanho da amostra do estudo foi definido por saturação do tempo durante o qual o questionário permaneceu disponibilizado para resposta online via web site, conforme pesquisa de Zebrack ${ }^{17}$. Sendo assim, o tamanho da amostra refere-se ao número de respondentes durante as dezoito semanas de coleta de dados.

Para a análise dos dados, foi empregada estatística descritiva básica, utilizando-se programa Microsoft Excel ${ }^{\circledR}$. As variáveis quantitativas foram divididas em intervalos e a frequência de respostas equivalente a cada um deles foi contabilizada. Para as variáveis qualitativas na forma de perguntas fechadas, foi utilizado cálculo de frequência de cada resposta. Já para as qualitativas, como perguntas abertas, as respostas foram primeiramente agrupadas conforme categorias e então contabilizada a frequência de resposta de cada uma delas. Em seguida, aplicou-se teste Qui-quadrado de Pearson para buscar associações entre as respostas.

\section{RES U L T A D O S}

Foram obtidas 445 respostas de nutricionistas, responsáveis por 413688 refeições diárias. Em sua maioria, as UPR funcionam de 5 a 7 dias por semana (99\%). Com relação ao porte, $47 \%$ servem até 300 refeições/dia (porte 1), $26 \%$ servem de 300 a 800 (porte 2) e 27\% servem 800 ou mais refeições diárias (porte 3).

Houve predominância de oferta de feijão carioca (48\%), seguida de feijão preto (36\%). Porém, enquanto nas UPR dos estados de São Paulo e Minas Gerais, o feijão predominantemente preparado é o carioca, nas do Rio Grande do Sul, Santa Catarina, Rio de Janeiro e Espírito Santo, o feijão preto é o mais oferecido. Já no
Paraná, 56\% dos estabelecimentos investigados oferecem feijão carioca e preto com mesma frequência na semana. Essa associação entre região e tipo de feijão consumido foi estatisticamente significante $(p<0,01)$.

Quanto ao remolho, 49\% relataram realizar o procedimento. Não foi encontrada associação significativa entre a realização de remolho e o tipo de feijão, porte da UPR, dias de funcionamento ou região do País.

Os motivos pelos quais o feijão é deixado ou não de remolho nos locais foram classificados em operacionais, nutricionais e sensoriais (Figura 2).

Os motivos referidos para realizar ou não o procedimento de remolho, bem como a combinação de razões apontadas, estão especificadas na Tabela 1, de acordo com o porte da UPR.

Dentre os locais que utilizam remolho, os motivos mais apontados são sensoriais e operacionais associados, independentemente do porte da UPR. Em segundo lugar, foram assinaladas razões estritamente operacionais em UPR de porte 1 e 2 e em UPR de porte 3, motivos operacionais, sensoriais e nutricionais associados. Porém, em relação aos estabelecimentos que não realizam o remolho, sobressai a questão operacional isoladamente, seguida do fato de nunca ter sido

\begin{tabular}{|c|c|c|}
\hline Operacionais & Nutricionais & Sensoriais \\
\hline $\begin{array}{l}\text { - Redução do tempo } \\
\text { de cocção com } \\
\text { remolho } \\
\text { - Falta de } \\
\text { equipamentos/ } \\
\text { utensílios/tempo } \\
\text { para realizar o } \\
\text { remolho } \\
\text { - Feijão cozinha fácil } \\
\text { não havendo } \\
\text { necessidade de } \\
\text { deixar de remolho } \\
\text { - Feijão necessita } \\
\text { ficar de remolho } \\
\text { em câmara fria, } \\
\text { mas não há espaço }\end{array}$ & $\begin{array}{l}\text { - Redução dos fatores } \\
\text { antinutricionais e } \\
\text { dos fatores } \\
\text { causadores de } \\
\text { flatulência com o } \\
\text { remolho } \\
\text {-Conservação dos } \\
\text { nutrientes sem } \\
\text { remolho }\end{array}$ & $\begin{array}{l}\text { - Melhor maciez e } \\
\text { melhor aparência } \\
\text { do caldo quando } \\
\text { deixado de } \\
\text { remolho } \\
\text { - Melhor textura e } \\
\text { sabor quando } \\
\text { não é deixado } \\
\text { de remolho }\end{array}$ \\
\hline
\end{tabular}

Figura 2. Razões para realização ou não do procedimento de remolho, conforme categorias. Florianópolis (SC), 2010. 
Tabela 1. Realização do procedimento de remolho nas Unidades Produtoras de Refeições do Sul e Sudeste do Brasil investigadas e o motivo de realização ou não desse procedimento de acordo com seu porte. Florianópolis (SC), 2010.

\begin{tabular}{|c|c|c|c|c|c|c|}
\hline \multirow{4}{*}{ Motivos } & \multicolumn{6}{|c|}{ Remolho (\%) } \\
\hline & \multicolumn{3}{|c|}{ Sim } & \multicolumn{3}{|c|}{ Não } \\
\hline & \multicolumn{3}{|c|}{ Porte } & \multicolumn{3}{|c|}{ Porte } \\
\hline & 1 & 2 & 3 & 1 & 2 & 3 \\
\hline Operacionais & 21 & 18 & 16 & 43 & 45 & 54 \\
\hline Sensoriais & 13 & 9 & 12 & 11 & 10 & 6 \\
\hline Nutricionais & 9 & 3 & 2 & 8 & 9 & 8 \\
\hline Nunca cogitou & 0 & 0 & 0 & 22 & 27 & 15 \\
\hline$O p^{*}+\operatorname{Sen} \neq$ & 28 & 38 & 44 & 3 & 5 & 3 \\
\hline $\mathrm{Op}^{*}+\mathrm{Nut}_{1}$ & 12 & 14 & 5 & 4 & 2 & 6 \\
\hline Sen $\neq+$ Nut & 5 & 7 & 3 & 7 & 2 & 5 \\
\hline $\mathrm{Op}^{*}+$ Senf+ Nut & 12 & 9 & 18 & 1 & 0 & 0 \\
\hline Outros motivos & 0 & 2 & 0 & 1 & 0 & 3 \\
\hline Total & 100 & 100 & 100 & 100 & 100 & 100 \\
\hline
\end{tabular}

Tabela 2. Tempo de remolho do feijão, segundo regiões e estados do Brasil onde estão localizadas as Unidades Produtoras de Refeição do Sul e Sudeste do Brasil investigadas. Florianópolis (SC), 2010.

\begin{tabular}{lccccc}
\hline \multirow{2}{*}{ Regiões e Estados } & \multicolumn{4}{c}{ Tempo de remolho (\%) } \\
\cline { 2 - 6 } & $<6 \mathrm{~h} 6-12 \mathrm{~h}$ & $>12 \mathrm{~h}$ & Total & $p$ \\
\hline Região Sul & 36 & 45 & 19 & $100 \%$ & \\
Rio Grande do Sul e Santa Catarina & 59 & 29 & 12 & $100 \%$ & \\
Paraná & & & & 0,192 \\
Região Sudeste & 51 & 37 & 12 & $100 \%$ \\
São Paulo & 55 & 42 & 3 & $100 \%$ \\
Rio de Janeiro e Espírito Santo & 67 & 25 & 8 & $100 \%$ \\
Minas Gerais & & & & \\
\hline
\end{tabular}

cogitada tal realização: ambos os motivos são independentes do porte da UPR.

Ainda entre UPR que realizam remolho, $49 \%$ deixam por até 6 horas; $39 \%$ deixam de 6 a 12 horas; $12 \%$ por mais de 12 horas. O porte da UPR não alterou a predominância de tempo de remolho. A maior parte dos estabelecimentos que deixam o feijão de remolho por menos de 6 horas (53\%) e de 6 a 12 horas (62\%) funciona sete dias por semana. Já a maioria dos que utilizam remolho de mais de 12 horas (54\%) atende cinco dias na semana. A relação entre tempo de remolho e região do Brasil onde as UPR se localizam está exposta na Tabela 2.
Nas UPR em que há maior consumo do feijão preto e nas localizadas no Rio Grande do Sul e Santa Catarina, observou-se maior frequência de remolho de 6 a 12 horas, porém não houve diferença estatística com relação aos demais estados.

Com relação a essas mesmas UPR que realizam remolho, quando perguntado se a água era descartada ou utilizada para a cocção, 31\% responderam que a água é utilizada e $69 \%$ que a água não é usada para cocção na sua UPR. Os motivos apontados foram classificados de forma semelhante aos apontados para realização ou não do remolho: operacionais (praticidade ao utilizar a água; costumes dos cozinheiros em descartar a água); sensoriais (preservar a cor e deixar o caldo mais grosso ao cozinhar com a água; eliminar a água escura e deixar o sabor mais agradável ao descartar a água); nutricionais (preservar nutrientes ao utilizar a água; diminuir antinutrientes e fatores de flatulência com o descarte). Dentre as UPR que descartam a água de remolho, foram relatados também motivos higiênico-sanitários, como a eliminação de sujidades e de organismo que possam eventualmente ter se proliferado na água.

Nos locais onde a água de remolho é utilizada, o motivo mais apontado é o nutricional $(41 \%)$, seguido do sensorial (25\%) e da associação entre nutricional e sensorial (25\%). Já onde há o descarte da água antes da cocção, as razões mais relatadas tiveram percentuais semelhantes entre si, sobressaindo, porém, a questão higiênico-sanitária (28\%), seguida das razões nutricionais (24\%) e da associação entre higiênico-sanitárias e nutricionais (22\%). Em resumo, as questões nutricionais e sensoriais parecem definir a utilização da água de remolho (91\%), e as questões higiênico-sanitárias e nutricionais parecem determinar o descarte da água (74\%). Entretanto, o porte da UPR parece influenciar a ordem em que esses motivos são apontados (Tabela 3).

As UPR de porte 1 utilizam a água de remolho para cocção, alegando como motivo principal a conservação dos nutrientes (55\%), en- 
Tabela 3. Utilização da água de remolho do feijão para cocção nas Unidades Produtoras de Refeição do Sul e Sudeste do Brasil investigadas e o motivo da utilização ou não da água, de acordo com seu porte. Florianópolis (SC), 2010.

\begin{tabular}{|c|c|c|c|c|c|c|}
\hline \multirow{4}{*}{ Motivos } & \multicolumn{6}{|c|}{ Utilização da água do remolho (\%) } \\
\hline & \multicolumn{3}{|c|}{ Sim } & \multicolumn{3}{|c|}{ Não } \\
\hline & \multicolumn{3}{|c|}{ Porte } & \multicolumn{3}{|c|}{ Porte } \\
\hline & 1 & 2 & 3 & 1 & 2 & 3 \\
\hline Operacionais & 10 & 6 & 0 & 11 & 15 & 9 \\
\hline Sensoriais & 24 & 35 & 18 & 0 & 0 & 0 \\
\hline Nutricionais & 55 & 29 & 32 & 24 & 20 & 29 \\
\hline Higiênico-sanitários & 0 & 0 & 0 & 32 & 25 & 24 \\
\hline$O p^{*}+$ Nut $\neq$ & 3 & 0 & 0 & 1 & 0 & 3 \\
\hline$O p^{*}+H i g \mid$ & 0 & 0 & 0 & 7 & 5 & 6 \\
\hline $\operatorname{Sen}^{* *}+\mathrm{Nut}_{1}^{1}$ & 7 & 29 & 45 & 0 & 0 & 0 \\
\hline $\mathrm{Nut}_{1}+\mathrm{Hig}$ & 0 & 0 & 0 & 19 & 28 & 24 \\
\hline $\mathrm{Op}^{*}+\mathrm{Sen}^{* *}+\mathrm{Higl}_{\mathrm{i}}^{\prime}$ & 0 & 0 & 0 & 1 & 3 & 0 \\
\hline $\mathrm{Op}^{*}+\mathrm{Nut}_{1}^{\prime}+\mathrm{Hig}_{1}^{1}$ & 0 & 0 & 0 & 4 & 5 & 6 \\
\hline Outros & 0 & 0 & 5 & 1 & 0 & 0 \\
\hline Total (\%) & 100 & 100 & 100 & 100 & 100 & 100 \\
\hline
\end{tabular}

Op*: Operacionais; Nutr!: Nutricionais; Higl: Higiênico-Sanitários; Sens ${ }^{* *}$ : Sensoriais.

quanto a questão sensorial (35\%) de conservação de cor e/ou textura do caldo é a mais apontada dentre as UPR de porte 2 . Já as UPR de porte 3 consideram a associação desses dois fatores (45\%) o motivo principal para o aproveitamento da água.

Dentre as que relataram descartar a água de remolho antes da cocção, as UPR de porte 1 declaram como principal motivo a questão higiênico-sanitária (32\%), ao passo que as de porte 2 colocam em primeiro a associação entre motivos higiênico-sanitários e nutricionais (28\%), e as de porte 3 consideram, primeiramente, a questão nutricional isolada (29\%).

Não foi possível aplicar teste Qui-quadrado sobre as razões apontadas para remolho ou descarte da água, uma vez que houve grande quantidade de categorias, bem como categorias diferentes entre as respostas "sim" e "não", o que poderia causar viés na análise.

\section{I S C U S S Ã O}

O número de respostas obtidas, 445 , foi considerado satisfatório, uma vez que a popu- lação do estudo limitou-se a nutricionistas atuantes nas regiões Sul e Sudeste do Brasil, na área de produção de refeições e que fossem responsáveis técnicos. No estudo de Akutsu ${ }^{18}$, cuja população abrangia todos os nutricionistas do Brasil, foram obtidas 587 respostas. Além disso, destaca-se que as respostas obtidas correspondem ao significativo valor de 413688 refeições diárias.

Os tipos de feijão mais oferecidos nas UPR investigadas estão de acordo com os dados de produção e consumo disponíveis, que indicam que o feijão carioca é o mais cultivado e consumido no Brasil, e que nos estados do Rio de Janeiro, Santa Catarina e Rio Grande do Sul, o feijão preto é o tipo preferido ${ }^{14}$.

A realização do procedimento de remolho do feijão parece ser de consenso entre diversos pesquisadores ${ }^{9-12}$; entretanto, nos locais investigados, observou-se que essa é ainda uma questão que divide as práticas. Apenas metade dos nutricionistas relatou realizar remolho na UPR, apesar de esse procedimento resultar no amaciamento da casca do grão e na economia de tempo e combustível na cocção, bem como causar redução de fatores antinutricionais e de flatulência ${ }^{9,19}$.

Uma vez que a região, o porte da UPR, o tipo de feijão e os dias de funcionamento não apareceram como determinantes na realização do remolho, observa-se que a execução dessa etapa pode estar mais relacionada à percepção do nutricionista ou de outros gestores da equipe da cozinha sobre sua importância.

Dentre os motivos apontados para realização do remolho no presente estudo, sobressaiuse a questão operacional associada à sensorial, referente à economia de tempo na cocção e ao amaciamento do grão, principalmente. Observa-se que, nos locais onde o remolho é realizado, há uma preocupação do nutricionista que extrapola a questão estritamente operacional, diferentemente dos locais onde não se realiza remolho, cujo motivo principal é apenas operacional, seguido do fato de nunca ter se cogitado 
realizá-lo. Assim, ressalta-se a importância de o nutricionista, por ser um profissional da saúde, refletir sobre seu papel na atuação em UPR, uma vez que as técnicas de preparo de alimentos podem ser determinantes na qualidade nutricional das preparações resultantes.

Ansaloni ${ }^{20}$, ao investigar a situação de trabalho de nutricionistas em UPR, observou que as decisões operacionais visando à questão econômica muitas vezes sobrepõem-se às decisões técnicas por ocasião das próprias condições de trabalho e exigências impostas aos cargos ocupados por esses nutricionistas.

Ademais, acredita-se que a carência de reflexão em torno do preparo do feijão possa também ser explicada por certa desvalorização cultural desse alimento. Segundo registros dos séculos XVIII e XIX, o feijão passou a ser visto como alimento de "pobre" e, muitas vezes, descrito como indigesto, desagradável e causador de moléstias ${ }^{21}$. A visão de que arroz e feijão era uma combinação fonte de proteínas para "pobres" 22 , no Brasil, passou a ter outro enfoque, oficialmente, após a adaptação da pirâmide alimentar para a realidade do país ${ }^{23}$ e a publicação do Guia Alimentar para a População Brasileira - $1^{\text {a }}$ versão em 2004, que recomendam o consumo do feijão para toda a população ${ }^{5}$. Entretanto, tais recomendações oficiais são recentes e não se dispõem de informações sobre a possível mudança desse enfoque a partir delas.

Com relação ao tempo de remolho, a maior parte dos respondentes declarou realizar remolho de até 6 horas; contudo, autores recomendam, no mínimo, 8 horas $^{24}$ ou 10 horas $^{19}$, quando utilizada água fria. Acredita-se que o remolho de, no mínimo, 12 horas seja mais realizado nos estabelecimentos que funcionam cinco dias na semana por haver, provavelmente, apenas um turno de trabalho nesses locais. Assim sendo, a decisão do tempo de remolho seria puramente operacional.

Na maior parte das UPR que realizam remolho, a água é descartada antes da cocção, fundamentalmente pela questão da eliminação de sujidades, micro-organismos, fatores antinutricionais e de flatulência. Essa prática é corroborada por estudos que analisaram o feijão cozido sem água de remolho, e que confirmam haver maior descarte de fatores antinutricionais ${ }^{9-11,25,26}$ e de flatulência ${ }^{27,28}$ quando a água de remolho é drenada, o que melhora a biodisponibilidade de nutrientes do feijão ${ }^{26,29}$. No entanto, deve-se ter cautela com a questão de eliminação de micro-organismos, pois, conforme Oliveira et al. ${ }^{30}$, a eliminação da água de remolho não melhorou a qualidade microbiológica do feijão processado.

Nas UPR que cozinham o feijão com a água de remolho, as principais razões relatadas são a preservação de coloração, textura do caldo e de nutrientes que se diluem nele, embora não tenham sido localizadas referências científicas que apoiem tal prática.

Quanto à perda de nutrientes com a eliminação da água de remolho, alguns estudos observaram que o conteúdo de minerais ${ }^{30,31}$ e de proteínas ${ }^{25,29}$ pode não se alterar com o descarte da água. Em outros casos, o descarte pode diminuir alguns macronutrientes ${ }^{10,11}$ e minerais ${ }^{26,32}$. Entretanto, mesmo havendo essa perda de minerais juntamente com o descarte da água do remolho, quando estudada a biodisponibilidade de minerais na preparação pronta, comparando as duas situações, observou-se que esta foi aumentada com relação às amostras sem descarte da água, provavelmente pela redução concomitante de fatores antinutricionais ${ }^{26}$.

Ademais, constatou-se que a cocção é a responsável pela maior perda de minerais durante o processamento do feijão, significativamente maior que a perda na água descartada ${ }^{27}$, mas há a possibilidade de consumo desses minerais pelo caldo $^{32}$, conforme o costume dos brasileiros. Contudo, não foram encontrados estudos avaliando vitaminas hidrossolúveis.

A ordem em que os motivos para descarte ou utilização da água são apontados neste estudo 
mostrou variar conforme o porte da UPR. Acredita-se que a maior preocupação em utilizar a água por conta da qualidade sensorial possa estar associada às UPR de porte 2 e 3 pela realização de compras em maiores volumes, que pode resultar em um maior tempo de estoque e, possivelmente, perda de atributos sensoriais com a diminuição de umidade do grão ${ }^{33}$.

Já o descarte da água, cujo motivo higiênico-sanitário está mais associado às UPR de porte 1 e 2, pode estar relacionado à preocupação com a temperatura em que o feijão é deixado de remolho, posto que há maior probabilidade de existir câmara fria nas UPR de maior porte.

\section{O N C L U S Ã O}

Observou-se que, em aproximadamente metade dos locais investigados, o remolho do feijão é realizado e que, na maioria desses locais, o tempo de remolho é de até 6 horas e a água de remolho é descartada antes da cocção.

A realização do procedimento de remolho do feijão parece ser determinada pela percepção do nutricionista sobre sua importância. A decisão sobre a não realização dessa etapa é determinada por motivos operacionais, em detrimento de nutricionais e sensoriais, os quais deveriam ser primordiais na atuação do nutricionista em UPR, uma vez que é um profissional da área da saúde.

Os resultados deste estudo podem refletir a escassez de discussões, durante a formação dos nutricionistas, quanto à influência das técnicas de preparo na qualidade nutricional e sensorial dos alimentos preparados, sobretudo quando se trata de feijão. Desse modo, considera-se importante essa discussão, que valoriza a importância das técnicas na qualidade das preparações para melhorar o esclarecimento de profissionais e alunos de nutrição.

Essa falta de reflexão sobre o preparo de feijão pode estar também relacionada à escassez de estudos conclusivos e concordantes sobre seu modo de preparo. O tema remolho do feijão care- ce de mais estudos devido à necessidade de avaliar a extensão das perdas de vitaminas hidrossolúveis nesse alimento com o descarte da água de remolho.

Assim, acredita-se haver necessidade de realização e melhor divulgação de estudos sobre técnicas de preparo do feijão a fim de aprimorar as condições nutricionais, sensoriais e higiênico-sanitárias na sua preparação.

\section{A GRADECIMENTOS}

À Coordenação de Aperfeiçoamento de Pessoal de Nível Superior pela concessão de bolsa de estudo, e aos Conselhos Regionais de Nutricionistas das regiões Sul e Sudeste do Brasil pela importante contribuição na coleta dos dados.

\section{COLABORADORES}

AC FERNANDES e RPC PROENÇA participaram da concepção do projeto, elaboração do instrumento de coleta, coleta e interpretação dos dados, redação e revisão do artigo. MCM CALVO participou da elaboração do instrumento de coleta, estruturação do banco de dados, análise estatística e interpretação dos dados e revisão do artigo.

\section{REFERÊ NCIAS}

1. Ornellas LH. A alimentação através dos tempos. $2^{\mathrm{a}}$ ed. Florianópolis: UFSC; 2000.

2. Organização das Nações Unidas para Agricultura e Alimentação. Base de dados estatísticos FAOSTAT [acesso 2008 out 14]. Disponível em: <http:// faostat.fao.org/>.

3. Instituto Brasileiro de Geografia e Estatística. Pesquisa de orçamentos familiares 2002-2003 e perfil das despesas: indicadores selecionados. [acesso 2008 out 14]. Disponível em: <http://www. sidra.ibge.gov.br/ bda/orcfam/default.asp?z=t\&o= 20\& $\mathrm{i}=\mathrm{P}>$.

4. Wander AE. Produção e consumo de feijão no Brasil, 1975-2005. Inf Econ. 2007; 37(2):7-21.

5. Brasil. Ministério da Saúde. Coordenação-Geral da Política de Alimentação e Nutrição. Guia alimentar 
para a população brasileira: promovendo a alimentação saudável. Brasília: Ministério da Saúde; 2006.

6. Rosa COB, Costa NMB, Leal PFG, Oliveira TT. Efeito do feijão preto (Phaseolus vulgaris L.) sem casca na redução do colesterol sangüíneo de ratos hipercolesterolêmicos. Arch Latinoam Nutr. 1998; 48(4): 299-305.

7. Olinto MT, Gigante D, Horta B, Silveira V, Oliveira I, Willett W. Major dietary patterns and cardiovascular risk factors among young Brazilian. Eur J Clin Nutr. 2011; 51(3):281-91. doi: 10.1007/s00394-011-0 213-4.

8. Silva MR, Silva MAAP. Aspectos nutricionais de fitatos e taninos. Rev Nutr. 1999; 12(1):21-32. doi: 10.1590/S1415-52731999000100003.

9. Oliveira AC, Carraro F, Reis SMPM, Ramos AG, Helbig E, Costa EL, et al. A eliminação da água não absorvida durante o remolho do feijão-comum aumentou o ganho de peso em ratos. Rev Nutr. 2001; 14(2):153-5. doi: 10.1590/S1415-527320 01000200009.

10. Oliveira AC, Queiroz KS, Helbig E. O processamento doméstico do feijão comum ocasionou uma redução nos fatores antinutricionais fitatos, taninos, no teor de amido e em fatores de flatulência rafinose, estaquiose e verbascose. Arch Latinoam Nutr. 2001; 51(3):276-83.

11. Ramirez-Cardenas L, Leonel AJ, Costa NMB. Efeito do processamento doméstico sobre o teor de nutrientes e de fatores antinutricionais de diferentes cultivares de feijão comum. Ciênc Tecnol Aliment. 2008; 28(1): 200-13. doi: 10.1590/S010 1-20612008000100029.

12. Fernandes AC, Nishida W, Proença RPC. Influence of soaking on the nutritional quality of common beans (Phaseolus vulgaris L.) cooked with or without the soaking water: a review. Int J Food Sci Technol. 2010; 45(11): 2209-18. doi: 10.1111/j.1 365-2621.2010.02395.x.

13. Brasil. Lei $n^{\circ} 8.234$, de 17 de setembro de 1991. Regulamenta a profissão de nutricionista e determina outras providências. Diário Oficial da União. 1991 set 18 [acesso 2010 jan 7]. Disponível em: $<$ http://www.cfn.org.br/ novosite/conteudo.aspx?! DMenu=56>.

14. Nascente AS, Diaz JLC, Del Peloso MJ, Faria LC, Melo LC, Costa JGC, et al. Atividades de pesquisa, desenvolvimento e transferência de tecnologia com o feijoeiro comum em São Paulo, Paraná e Santa Catarina (2004/2005). Santo Antônio de Goiás: Embrapa Arroz e Feijão; 2005 [acesso 2010 jan 3]. Disponível em: <www.cnpaf.embrapa.br>.
15. Évora YDM. As possibilidades de uso da internet na pesquisa em enfermagem. Rev Eletrônica Enferm. 2004; 6(3):395-9 [acesso 2008 out 27]. Disponível em: <www.fen.ufg.br>.

16. Rea LM, Parker R. Metodologia da pesquisa: do planejamento à execução. São Paulo: Pioneira Thomson Learning; 2002.

17. Zebrack B. Information and service needs for young adult cancer patients. Support Care Cancer. 2008; 16(12):1353-60. doi: 10.1007/s00520-008-0435-z.

18. Akutsu RC. Brazilian dieticians: professional and demographic profiles. Rev Nutr. 2008; 21(1):7-19. doi: 10.1590/S1415-52732008000100002.

19. Botelho RA, Araújo WMC, Zandonadi RP, Ramos KL. Transformação dos alimentos: cereais e leguminosas. In: Araújo WMC, Montebello NP, Botelho RA, Borgo LA. Alquimia dos alimentos. Brasília: Senac; 2007.

20. Ansaloni JA. Situação de trabalho dos nutricionistas em empresas de refeições coletivas de Minas Gerais: trabalho técnico, supervisão ou gerência? Rev Nutr. 1999; 12(3):241-60. doi: 10.1590/\$1415-52731 999000300005.

21. Cascudo LC. História da alimentação no Brasil. $3^{a}$ ed. São Paulo: Global; 2004.

22. Maciel ME, Menasche, R. Alimentação e cultura, identidade e cidadania: você tem fome de quê? Rev Democracia Viva [Internet]. 2003 [acesso 2010 jan 5]; 1(16):3-4. Disponível em: <http://www. ibase.br/pt/biblioteca-2/>.

23. Philippi ST, Latterza AR, Cruz ATR, Ribeiro LC. (1999). Pirâmide alimentar adaptada: guia para escolha dos alimentos. Rev Nutr. 1999; 12(1): 65-80. doi: 10.1590/S1415-52731999000100006.

24. Wright J, Treuille E. Le cordon bleu: todas as técnicas culinárias. São Paulo: Editora Marco Zero; 1997.

25. Toledo TCF, Canniatti-Brazaca SG. Chemical and nutritional evaluation of Carioca beans (Phaseolus vulgaris L.) cooked by different methods. Ciênc Tecnol Aliment. 2008; 28(2):355-60. doi: 10.159 0/S0101-20612008000200013.

26. ElMaki HB, Rahaman SMA, Idris WH, Hassan $A B$, Babiker EE, ElTinay AH. Content of antinutritional factors and $\mathrm{HCl}$-extractability of minerals from white bean (Phaseolus vulgaris) cultivars: influence of soaking and/or cooking. Food Chem. 2007; 100(1):362-8. doi: 10.1016/j.foodchem.2005. 09.060 .

27. Granito M, Brito Y, Torres A. Chemical composition, antioxidant capacity and functionality of raw and processed Phaseolus lunatus. J Sci Food Agric. 2007; 87(15):2801-09. 
28. Shimelis EA, Rakshit SK. Effect of processing on antinutrients and in vitro protein digestibility of kidney bean (Phaseolus vulgaris L.) varieties grown in East Africa. Food Chem. 2007; 103(1):161-72. doi: 10.1016/j.foodchem. 2006.08.005.

29. Rehman Z-U, Shah WH. Domestic processing effects on some insoluble dietary fibre components of various food legumes. Food Chem. 2004; 87(4): 613-7. doi: 10.1016/j.foodchem.2004.01.012

30. Oliveira VR, Ribeiro ND, Jost E, Londero PMG. Nutritional and microbiological quality of common beans (Phaseolus vulgaris L.) cooked with or without the use of soaking water. Ciênc Agrotec. 2008; 32(6):1912-8. doi: 10.1590/\$1413-70542 008000600034.

31. Chopra S, Sankhala A. Effect of soaking and sprouting on tannin, phytate and in vitro iron in underutilized legumes - Horse gram (Dolichos biflorus) and moth bean (Phaseolus aconitifolius). J Food Sci Technol. 2004; 41(5):547-50.

32. Huma N, Anjum FM, Sehar S, Khan MI, Hussain S. Effect of soaking and cooking on nutritional quality and safety of legumes. Nutr Food Sci. 2008; 38 (6):570-7. doi: 10.1108/00346650810920187.

33. Ribeiro HJSS, Prudêncio-Junqueira SH, Miaguy TM. Propriedades físicas e químicas de feijão comum preto, cultivar lapar 44, após envelhecimento acelerado. Ciênc Tecnol Aliment. 2005; 25(1):165-9.

Recebido em: 6/4/2011

Versão final em: 21/11/2011

Aprovado em: 9/1/2012 
